\title{
Overlap syndromes in systemic sclerosis
}

\author{
Ewa Wielosz, Maria Majdan, Magdalena Dryglewska, Bożena Targońska-Stępniak
}

Department of Rheumatology and Connective Tissue Diseases, Medical University of Lublin, Lublin, Poland

Adv Dermatol Allergol 2018; XXXV (3): 246-250

DOI: https://doi.org/10.5114/ada.2018.72662

\begin{abstract}
Introduction: It is known, that course of the disease differs between overlap syndromes (OS) and systemic sclerosis (SSc) group.

Aim: To compare the prevalence of OS in limited cutaneous systemic sclerosis (IcSSc) and diffuse cutaneous SSc (dcSSc) and to analyze the presence of different manifestations in the SSc and OS group.

Material and methods: The study included 126 European Caucasian SSc patients ( 99 females and 27 males) hospitalized consecutively in the Department of Rheumatology and Connective Tissue Diseases. Patients fulfilled the American College of Rheumatology (ACR) classification criteria of SSc (57 - dcSSc and $69-$ IcSSc). The study groups were determined according to the subtype of SSc, coexistence of other connective tissue diseases (CTDs), and incidence of clinical and serological manifestations.

Results: In our SSc study group, 28/126 patients (22\%) were affected by more than one CTD. The prevalence of OS was significantly higher in the IcSSc group - 33\% (23/69) compared to the dcSSc group - 8\% (5/57). We found that mortality and digital ulcers were significantly higher, whereas kidney involvement and arthritis were significantly lower in the SSc group compared to the OS group. The prevalence of anti-topoisomerase I (a-Scl-70) was significantly higher, and prevalence of anti-PM/Scl, anti-Ro-52 antibodies was significantly lower in the SSc group compared to the OS group.

Conclusions: Overlap syndromes were more common in lcSSc than in dcSSc. The course of the disorder and internal organ involvement were different in OS compared to SSc patients.
\end{abstract}

Key words: systemic sclerosis, overlap syndrome.

\section{Introduction}

Overlap syndromes (OS) are autoimmune disorders in which classification criteria of at least two connective tissue diseases (CTDs) are fulfilled. The most common combinations are systemic sclerosis (SSC) and Sjögren syndrome (SS), dermatomyositis (DM) or polymyositis (PM), rheumatoid arthritis (RA), systemic lupus erythematosus (SLE) [1-4]. Occasionally, three or more CTDs overlap or other non-rheumatic autoimmune diseases occur, such as autoimmune thyroiditis (AT), autoimmune hepatitis, primary biliary cirrhosis (PBC), as well as autoimmune thrombocytopenia, coeliac disease or vitiligo [2, $3,5]$. In the course of SSc, $20-30 \%$ of patients develop OS [1-3]. The clinical presentation of OS patients is very heterogeneous and that diversity depends on the kind and subtype of diseases that overlap [6]. According to LeRoy et al., there are two different subtypes of SSC - limited cutaneous SSc (IcSSc) and diffuse cutaneous SSc (dcSSc) [7]. The course of the disease and prevalence of organ involvement differs in SSC and in OS. Moreover, there are some data describing differences in the prevalence of OS between the two subtypes of SSc [6].

\section{Aim}

The aim of the present study was to compare the prevalence of OS in patients with IcSSc and dcSSc, as well as to compare different manifestations of SSc in patients with SSC and with OS.

\section{Material and methods}

The study included 126 (99 female and 27 male) European Caucasian SSc patients hospitalized consecutively in the Department of Rheumatology and Connective Tissue Diseases, Medical University of Lublin. All patients provided written informed consent to participate in the study according to the Declaration of Helsinki. The study design was approved by the ethical committee. All patients fulfilled the American College of Rheumatology (ACR)/European League Against Rheumatism (EULAR) classification 
criteria of SSc [8]. According to LeRoy et al., patients were classified as IcSSc or dcSSc [7]. Limited cutaneous SSc means that only limited areas of the skin are thick; the distal parts of the upper and lower limbs below, but not above, the elbows and knees with or without involvement of the face. The dcSSc type was recognized in 57 patients and IcSSc in 69 patients. The coexistence of other CTDS was analyzed in lcSSc and dcSSc patients. Moreover, patients were divided into two groups: SSC and OS (Table 1). The subtype of SSc, incidence of internal organ involvement, prevalence of death and serological profile were determined in the SSC and OS groups. Patients with more than one CTD were classified as OS general. Rheumatoid arthritis and SS were diagnosed according to the new ACR/EULAR classification criteria $[9,10]$. Systemic lupus erythematosus was diagnosed according to 2012 SLICC classification criteria [11]. Antiphospholipid syndrome (APS) was diagnosed according to the original 1999 APS (Sapporo) classification criteria and 2006 APS criteria revised in Sydney [12]. Polymyositis/dermatomyositis was diagnosed according to the Bohan and Peter criteria [13]. Two other autoimmune diseases, AT and PBC, were diagnosed on the basis of serological and histological criteria. Organ involvement was assessed according to the clinical symptoms and the results of diagnostic tests. Interstitial lung disease (ILD) was defined as a "ground glass" pattern or bibasilar pulmonary fibrosis revealed on a high-resolution computer tomography (HRCT) scan. The DLCO test (\% predicted diffusing capacity for carbon monoxide) and total lung capacity (TLC) were performed to assess the pulmonary function. Heart involvement was established as arrhythmia, conduction disturbances or heart failure. Pulmonary arterial hypertension (PAH) was defined as systolic pulmonary arterial pressure (SPAP) > $35 \mathrm{~mm} \mathrm{Hg}$ in Doppler echocardiography and was determined only at rest. Myalgia or myositis was defined as pain or weakness of muscles and/or increased serum creatine phosphokinase (CPK) concentration. Joint involvement was determined as joint tenderness and swelling. Gastrointestinal tract involvement was defined according to clinical symptoms such as dysphagia, heartburn, diarrhoea or bloating and was examined by a barium swallow. Renal involvement was defined as the development of scleroderma renal crisis (SRC) or presence of proteinuria and elevated serum creatinine concentration. Calcinosis and digital erosions were also assessed. Serum samples were obtained from each patient. Moreover, presence of antibodies was assessed in the study groups, applying the commercial test - EUROLINE Systemic Sclerosis Profile, which is used to determine antibodies directed against SSc-specific antigens, such as anti-topoisomerase I (anti-Scl-70) and anticentromere autoantibodies (ACAs), anti-RNA polymerase III (anti-RNA pol III), as well as more rare anti-PM/SCl, anti-Ku, anti-Th/To anti-Ro52 and autoantibodies against nucleolus-organizing region-90 (anti-NOR90). Detection and interpretation of results was carried out electronically using the specific program Euroimmun - EUROLINE Scan. Sera were tested for myeloperoxidase (MPO) or proteinase 3 (PR3) anti-neutrophil cytoplasmic antibodies (ANCA) by the ELISA test.

\section{Statistical analysis}

The statistical analysis was performed with Statistica 10.0. Data were analysed using the $\chi^{2}$ test (variation test) for comparison between groups. $P$-value $<0.05$ was considered as statistically significant.

\section{Results}

According to our observations, 28/126 (22\%) patients were affected by more than one CTDs. The time from the onset of Raynaud's phenomenon to the diagnosis was significantly longer in ICSSc when compared with the dcSSc group $(8.0 \pm 7.3$ years $(0.5-30)$ vs. $3.0 \pm 3.2$ years $(0.5-15) ; p<0.001)$. The prevalence of OS was significantly higher in IcSSc when compared with dcSSc (33\% (23/69) vs. $8 \%$ (5/57) patients; $p=0.0007)$. Moreover, the incidence of IcSSC was significantly higher in the OS group, as compared with the SSc group without other CTDs (82\% vs. $47 \%, p=0.001$ ) (Figure 1 ).

The prevalence of an additional CTD in the entire group and in the OS group was as follows: SSC/SS - 9 pa-

Table 1. Characteristics of the systemic sclerosis and overlap syndrome group

\begin{tabular}{lcc}
\hline Parameter & Systemic sclerosis & Overlap syndromes (systemic sclerosis/connective tissue diseases) \\
\hline Number of patients: & 98 & 28 \\
\hline dcSSc & 52 & 25 \\
\hline IcSSC & 46 & 23 \\
\hline Gender: & 75 & 24 \\
\hline Female & 23 & 4 \\
\hline Male & $53.5 \pm 13.9(18.0-81.0)$ & $54.1 \pm 10.4(22.0-71.0)$ \\
\hline Age [years] & $6.3 \pm 6.1(0.0-23.0)$ & $6.1 \pm 5.8(0.0-22.0)$ \\
\hline $\begin{array}{l}\text { Duration of disease [years] } \\
\text { Time from onset of Raynaud's } \\
\text { phenomenon to diagnosis [years] }\end{array}$ & $5.6 \pm 6.2(0.3-30.0)$ & $6.2 \pm 4.8(1.0-20.0)$ \\
\hline
\end{tabular}

Data were presented as number. 


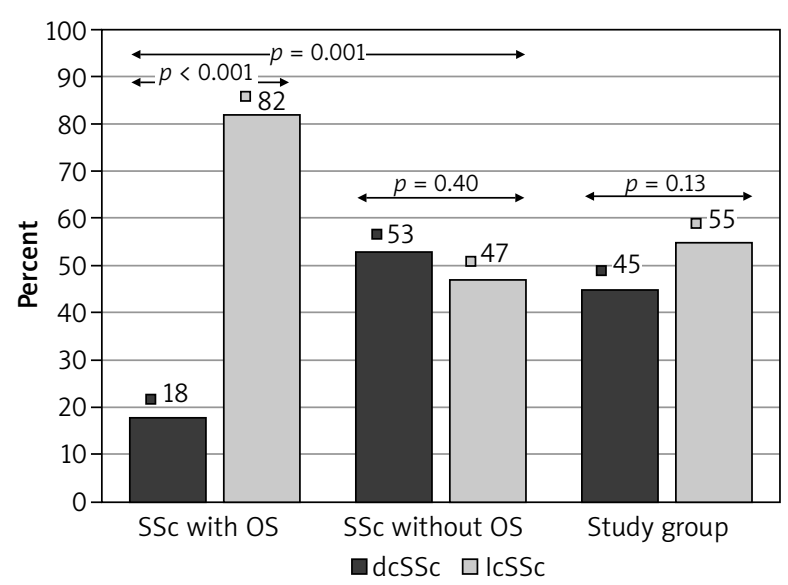

Figure 1. Comparison of dcSSc and IcSSc prevalence between group SSc and group OS

tients $(7.1 \%, 32.1 \%$ ) (2 patients dcSSc/SS and 7 patients IcSSC/SS); SSc/RA coexisted in 8 patients $(6.3 \%, 26.6 \%)$ and all patients had ICSSC/RA; SSC/PM was found in 7 cases (5.5\%, 25\%) (5 patients IcSSc/PM and 2 dcSSc/ PM); SSc/SLE coexisted in 2 patients (1.6\%, 7.1\%) and SSC/APS in another 2 cases (1.6\%, 7.1\%). Five patients developed more than two CTDs (3.9\%, 17.8\%).

In our study group, it was found that mortality and presence of digital ulcers was significantly higher in the SSc group in comparison with the OS group (21.4\% vs. $3.6 \%$ respectively, $p=0.043$ and $21.4 \%$ vs. $3.6 \%$ respectively, $p=0.043)$. On the other hand, the prevalence of kidney involvement and arthritis was significantly higher in the OS group in comparison with the SSc group $(67.8 \%$ vs. $29.9 \%$ respectively, $p=0.003$ and $68 \%$ vs. $20 \%$ respectively, $p<0.001)$. There were no significant differences in the prevalence of lung, heart, gastrointestinal or muscle involvement between SSc and OS groups.

The incidence of anti-Scl-70 antibodies was significantly higher in the SSC group in comparison with the OS group (43\% vs. 14\%, $p=0.005)$; however, the incidence of other antibodies (anti-PM/Scl and anti-Ro-52) was significantly lower in the SSc group in comparison with the OS group (10\% vs. $25 \%$ respectively, $p=0.039$ and $21 \%$ vs. $42 \%$ respectively, $p=0.024)$. No significant differences in the prevalence of ACAs, anti-RNA pol III, anti Th1/Th0, anti-Ku and anti-NOR 90 antibodies were found between the study groups (Table 2). In our study group, there were no patients with the presence of ANCA.

Considering organ-specific autoimmune diseases we found that 30/126 patients (23\%) had AT and 10/126 patients (8\%) developed PBC. There were no significant differences in the prevalence of AT in the SSc group when compared with the SSc/CTDs group (21/98 (21\%) vs. 9/28 (32\%), NS). In AT, $12 / 30$ patients had dcSSc and 18/30 patients had IcSSc. Interestingly, the prevalence of PBC was markedly lower in the SSc group when compared with the SSc/CTD group (5/98 (5\%) vs. 5/28 (17\%), $p=0.04$ ). Additionally we observed that the incidence of PBC was significantly higher in IcSSc than in dcSSC (8/10 patients had IcSSc and 2/10 patients had dcSSc, $p=0.01$ ).

\section{Discussion}

In our study group of 126 patients with SSc, 28 (22\%) presented features of OS. The prevalence of OS was found to be significantly higher in patients with ICSSC than in patients with dcSSc. Concurrently, the incidence of IcSSc was significantly higher in OS than in SSc patients with no other CTDs.

In the OS group, the most prevalent concomitant CTDs included SS, RA and PM. Overlap of SSC and SLE or APS was quite rare. Results of our study indicate that SSC/PM seems to be the most severe type of OS. In our SSc/PM group, 6/7 patients developed gastrointestinal tract involvement and ILD, as well as 2/7 deaths occurred in that group. Additionally in our SSc patients we found symptoms of AT and PBC, with more common IcSSc subtype, both in PBC and AT.

The comparison of patients with OS and SSc (with no concomitant (TD) revealed a significantly higher prevalence of kidney involvement and arthritis, as well as significantly lower digital ulcers and mortality rate in the OS group, suggesting a more favourable course of the disease in OS patients. It is likely that a lower mortality rate in the OS group was also associated with a less severe course of IcSSc, which occurred more often in OS patients.

In serological tests, in patients with OS the prevalence of anti Ro-52 and anti-PM/Scl was significantly higher and prevalence of anti-Scl-70 significantly lower, when compared with SSc patients (with no concomitant CTD). There was no significant difference in the presence of other assessed antibodies. Anti-Scl-70 are the most typical antibodies for SSc. As is well known, anti-Ro and anti-La antibodies are specific for SS. Anti-Ro-52 and anti$\mathrm{PM} / \mathrm{Scl}$ antibodies may occur in SSc, however they are usually detected in other CTDS such as SS, SLE that could explain their prevalence in OS patients.

The data from the literature are similar to our results. Balbir-Gurman and Braun-Mascovici demonstrated that 40/165 (24.2\%) SSc patients fulfilled the criteria of OS [1]. According to Caramaschi et al., 32.2\% of cases showed one autoimmune disease in association with SSc [2]. In another large study, 332 out of 1700 patients with SSc (20\%) had OS [3]. Reports considering the prevalence of SSC subtypes in OS are diverse. Some studies reported that dcSSc seemed to be more prevalent in SSC/PM (47.4\%) in comparison with other OS. However most patients with SSc/SS had IcSSC [1] and the vast majority of SSc/RA patients suffered from IcSSc [14]. According to the large study including 123 SSc/RA patients, 81.2\% of patients were af- 
Table 2. Comparison of the prevalence of internal organ involvement and selected antibodies between the systemic sclerosis groups "without" and "with" overlap syndrome

\begin{tabular}{|c|c|c|c|}
\hline Clinical markers & Systemic sclerosis & $\begin{array}{c}\text { Overlap syndromes } \\
\text { (systemic sclerosis/connective tissue diseases) }\end{array}$ & $P$-value \\
\hline Scleroderma renal crisis & $6(6 \%)$ & $1(4 \%)$ & 0.6 \\
\hline Kidney involvement & $29(30 \%)$ & $19(68 \%)$ & 0.003 \\
\hline Decreased DLCO & $53(58 \%)$ & $13(46 \%)$ & 0.3 \\
\hline Decreased TLC & $27(27 \%)$ & $4(14 \%)$ & 0.1 \\
\hline Pulmonary arterial hypertension (echo) & $26(27 \%)$ & $4(14 \%)$ & 0.2 \\
\hline Gastrointestinal tract involvement & $59(63 \%)$ & $17(61 \%)$ & 0.8 \\
\hline ILD (HRCT) & $60(61 \%)$ & $13(46 \%)$ & 0.2 \\
\hline Heart involvement & $38(39 \%)$ & $8(29 \%)$ & 0.3 \\
\hline Arterial hypertension & $43(44 \%)$ & $11(39 \%)$ & 0.6 \\
\hline Calcinosis & $17(21 \%)$ & $6(21 \%)$ & 0.9 \\
\hline Arthritis & $19(20 \%)$ & $19(68 \%)$ & $<0.001$ \\
\hline Arthralgia & $80(84 \%)$ & $27(96 \%)$ & 0.09 \\
\hline Myalgia & $17(18 \%)$ & $8(29 \%)$ & 0.2 \\
\hline Digital ulcers & $21(21 \%)$ & $4(4 \%)$ & 0.04 \\
\hline Death & $21(21 \%)$ & $4(4 \%)$ & 0.04 \\
\hline ACAs & $18 / 98(18 \%)$ & $6 / 28(21 \%)$ & 0.7 \\
\hline Anti-Scl 70 & 43/98 (43\%) & $4 / 28(14 \%)$ & 0.005 \\
\hline Anti-RNA polymerase III & $8 / 98(8 \%)$ & $2 / 28(7 \%)$ & 0.3 \\
\hline Anti-Ku & $3 / 98(3 \%)$ & $1 / 28(3 \%)$ & 1.0 \\
\hline Anti-Pm/Scl & $10 / 98(10 \%)$ & $7 / 28(25 \%)$ & 0.04 \\
\hline Anti-Ro 52 & $21 / 98(21 \%)$ & $12 / 28(43 \%)$ & 0.02 \\
\hline Anti-Th/To & $2 / 98(2 \%)$ & $0 / 28(0 \%)$ & 0.4 \\
\hline Anti-NOR90 & 3/98 (3\%) & $2 / 28$ (7\%) & 0.3 \\
\hline
\end{tabular}

Data were presented as numbers and percentages. P-value of $<0.05$ was considered statistically significant.

fected by IcSSc and $17.9 \%$ by dcSSc [3]. Our results are comparable to the data from the literature regarding SSc/SS and SSc/RA groups. In our study, all patients with SSC/RA had the IcSSc subtype and $78 \%$ of cases with $\mathrm{SSc}$ SS had ICSSC. The results of our observations in the SSc/PM group differ from those in the literature. Most of our patients in the SSC/PM group developed IcSSc subtype.

Data from the literature indicate that SSc/myositis and SSC/SS were the most common combinations. Furthermore, the clinical course of SSc/SS was reported to be less severe in comparison with SSc without SS. Simultaneously, SSc/PM was found to have a more severe course of the disease with potentially higher morbidity and mortality [1]. Systemic sclerosis/polymyositis was associated with digital ulcers, gastrointestinal tract involvement, ILD, arthritis and cardiomyopathy $[1,3,14]$. According to the literature, overlaps of SSC and SLE or SSc/APS were quite rare combinations, often associated with an uncontrolled course and even a fatal out- come [1]. The prevalence of SSc/APS ranges from $7 \%$ to $13 \%[15]$.

Some studies compared clinical and serological parameters in SSc and OS groups. According to one study, fewer patients with OS suffered from digital ulcers, as compared with SSc patients (18.2\% vs. 33.3\%, $p<0.0001$ ) [6]. According to our findings, the incidence of digital ulcers was higher in the SSc group than in the OS group. Interestingly, the incidence of kidney involvement was found to be lower in the SSc group compared to the OS group. An explanation of the result is quite difficult. The hypothesis that a more prevalent kidney involvement in the OS group could be associated with typical renal manifestations of other CTDs (e.g. lupus nephritis, Sjögren's syndrome-associated interstitial nephritis, or vasculitis) seems too weak. In our study group there were no patients with SSc/ANCA-associated vasculitis and the prevalence of SLE was very low. Moreover, arthritis was observed most commonly in the OS group. We take into consideration the fact that patients with 
OS use NSAIDs quite frequently due to arthritis and that could aggravate renal function. Our results are similar to data in the literature. According to one study, musculoskeletal involvement was the most frequent organ manifestation in OS [16].

According to the literature, the prevalence of AT in SSc was $14-30 \%$ and mostly accompanied IcSSC [2, 17], whereas PBC occurred in about $15 \%$ of SSc cases, most commonly in IcSSc subtype $[1,5]$. Our results are similar.

The serological results of our study are consistent with data in the literature. In the large cohort, Pakozdi et al. demonstrated that patients SSC/PM or DM carried anti-PM/SCl antibodies in 33.1\% of cases [3]. According to this study, anti-Ro/SSA and anti-La/SSB antibodies were reported in $38.8 \% \mathrm{SSC}$ SS cases [3]. In a large German cohort, the prevalence of ACAs was similar (37.3\% vs. 36.4\%), but prevalence of anti Scl-70 antibodies was lower in SSc/SS than in SSc patients [16]. Interestingly, anti-RNA Pol III antibodies were infrequently observed in SSc/SS compared to SSc [3].

The limitations of our study include a small number of patients in the different subtypes of OS as well as lack of follow-up observation.

\section{Conclusions}

In the entire group of patients with SSc, the prevalence of OS was significantly higher in patients with IcSSc than in patients with dcSSc. Moreover, the clinical symptoms and course of the disease as well as serological markers differed between OS and SSc groups [18-20]. The findings of our study and data in the literature suggest that patients with OS should be carefully evaluated and considered as a separate subset of patients with SSc.

\section{Conflict of interest}

The authors declare no conflict of interest.

\section{References}

1. Balbir-Gurman A, Braun-Moscovici Y. Scleroderma overlap syndrome. Isr Med Assoc J 2011; 13: 14-20.

2. Caramaschi P, Biasi D, Volpe A, et al. Coexistence of systemic sclerosis with other autoimmune diseases. Rheumatol Int 2007; 27: 407-10.

3. Pakozdi A, Nihtyanova S, Moinzadeh P, et al. Clinical and serological hallmarks of systemic sclerosis overlap syndromes. J Rheumatol 2011; 38: 2406-9.

4. Aguila LA, Lopes MR, Pretti FZ, et al. Clinical and laboratory features of overlap syndromes of idiopathic inflammatory myopathies associated with systemic lupus erythematosus, systemic sclerosis, or rheumatoid arthritis. Clin Rheumatol 2014; 33: 1093-8.

5. Imura-Kumada S, Hasegawa M, Matsushita T, et al. High prevalence of primary biliary cirrhosis and disease-associated autoantibodies in Japanese patients with systemic sclerosis. Mod Rheumatol 2012; 22: 892-8.
6. Moinzadeh P, Aberer E, Ahmadi-Simab K, et al. Disease progression in systemic sclerosis-overlap syndrome is significantly different from limited and diffuse cutaneous systemic sclerosis. Ann Rheum Dis 2015; 74: 730-7.

7. LeRoy EC, Black C, Fleishmajer R. Scleroderma (systemic sclerosis): classification, subsets and pathogenesis. J Rheumatol 1988; 15: 202-4.

8. van den Hoogen F, Khanna D, Fransen J, et al. 2013 classification criteria for systemic sclerosis: an American College of Rheumatology/European League against Rheumatism Collaborative Initiative. Ann Rheum Dis 2013; 72: 1747-55.

9. Aletaha D, Neogi T, Silman AJ, et al. 2010 rheumatoid arthritis classification criteria: an American College of Rheumatology/European League Against Rheumatism collaborative initiative. Ann Rheum Dis 2010; 69: 1580-8.

10. Shiboski CH, Shiboski SC, Seror R, et al. 2016 American College of Rheumatology/European League Against Rheumatism classification criteria for primary Sjögren's syndrome: a consensus and data-driven methodology involving three international patient cohorts. Arthritis Rheumatol 2017; 69: 35-45.

11. Petri M, Orbai AM, Alarcón GS, et al. Derivation and validation of the systemic lupus international collaborating clinics classification criteria for systemic lupus erythematosus. Arthritis Rheum 2012; 64: 2677-86.

12. Miyakis S, Lockshin MD, Atsumi T, et al. International consensus statement on an update of the classification criteria for definite antiphospholipid syndrome (APS). J Thromb Haemost 2006; 4: 295-306.

13. Bohan A, Peter JB. Polymyositis and dermatomyositis (first of two parts). N Engl J Med 1975; 292: 344-7; 403-7.

14. Iaccarino L, Gatto M, Bettio S, et al. Overlap connective tissue disease syndromes. Autoimmun Rev 2013; 12: 363-73.

15. Wielosz E, Dryglewska M, Majdan M. Antiphospholipid antibodies and kidney involvement in patients with systemic sclerosis. Clin Rheumatol 2009; 28: 955-9.

16. Hunzelmann N, Genth E, Krieg T, et al. The registry of the German Network for Systemic Scleroderma: frequency of disease subsets and patterns of organ involvement. Rheumatology (Oxford) 2008; 47: 1185-92.

17. Biró E, Szekanecz Z, Czirják L, et al. Association of systemic and thyroid autoimmune diseases. Clin Rheumatol 2006; 25: 240-5.

18. Wielosz E, Majdan M, Zychowska I, Jeleniewicz R. Coexistence of five autoimmune diseases: diagnostic and therapeutic difficulties. Rheumatol Int 2008; 28: 919-23.

19. Hervier B, Lambert M, Hachulla E, et al. Anti-synthetase syndrome positive for anti-isoleucyl-tRNA synthetase antibodies: an unusual case overlapping with systemic sclerosis and Sjogren's syndrome. Rheumatology (Oxford) 2011; 50: $1175-6$.

20. Szücs G, Szekanecz Z, Zilahi E, et al. Systemic sclerosis-rheumatoid arthritis overlap syndrome: a unique combination of features suggests a distinct genetic, serological and clinical entity. Rheumatology (Oxford) 2007; 46: 989-3. 